\title{
Mitigating Implicit Bias in Patient-Clinician Communication in Clinical Encounters: Early Insights from the COmmuNity-engaged SimULation Training (CONSULT) Trial
}

Jennifer Tjia ( $\square$ Jennifer.tjia@umassmed.edu )

University of Massachusetts Medical School https://orcid.org/0000-0003-4541-0460

Michele Pugnaire

University of Massachusetts Medical School

Joanne Calista

Center for Health Impact

Nancy Esparza

Center for Health Impact

Olga Valdman

University of Massachusetts Medical School

Maria Garcia

University of Massachusetts Medical School

Majid Yazdani

University of Massachusetts Medical School

Janet Hale

University of Massachusetts Medical School

Jill Terrien

University of Massachusetts Medical School

\section{Ethan Eisdorfer}

University of Massachusetts Medical School

Valerie Zolezzi-Wyndham

Promoting Good

Germán Chiriboga

University of Massachusetts Medical School

Lynley Rappaport

University of Massachusetts Medical School

Geraldine Puerto

University of Massachusetts Medical School

Elizabeth Dykhouse

University of Massachusetts Medical School 


\section{Stacy Potts}

University of Massachusetts Medical School Andriana Foiles Sifuentes

University of Massachusetts Medical School

\section{Sylvia Stanhope}

University of Massachusetts Medical School

Jeroan Allison

University of Massachusetts Medical School

Vennesa Duodu

University of Massachusetts Medical School

Janice Sabin

University of Washington

\section{Research article}

Keywords: COmmuNity-engaged SimULation Training, healthcare disparities, bias awareness, communication skills, implicit bias

Posted Date: July 8th, 2020

DOI: https://doi.org/10.21203/rs.3.rs-39615/v1

License: (c) (i) This work is licensed under a Creative Commons Attribution 4.0 International License. Read Full License 


\section{Abstract}

\section{Background}

To address the gap in knowledge about how to design feasible and acceptable trainings for clinicians that aim to mitigate implicit bias in clinical encounters, we report the early insights from the COmmuNityengaged SimULation Training for Blood Pressure Control (CONSULT) Trial.

\section{Methods}

We engaged academic and community stakeholders to design, pilot test and implement a training program addressing healthcare disparities knowledge, bias awareness, and communication skills focused on bias mitigation. A stepped wedge cluster randomized trial was developed to determine intervention dose effects. We assessed the CONSULT training program through structured feedback using online surveys, real-time comments, and individualized feedback from trainees, faculty and standardized patients.

Results

The first training cohort completed the intervention $(\mathrm{N}=64)$. Feedback prompted training program revisions as follows: reducing overall time burden and the number of implicit bias assessments; supplementing on-line learning with augmented in-person interactive sessions. Feedback also reinforced the critical importance of having highly skilled facilitators versed in implicit bias.

\section{Conclusions}

Iterative stakeholder engagement is essential for developing and revising educational interventions aimed at raising bias awareness and mitigating the effects of implicit bias.

Trial Registration

ClinicalTrials.gov, NCT 03375918. Registered December 18, 2017, https://clinicaltrials.gov/ct2/show/NCT03375918?id=NCT+03375918\&draw=2\&rank=1

\section{Background}

Evidence suggests that healthcare professionals have negative explicit and implicit biases toward minority and poor patients, ${ }^{1,2}$ which can adversely affect clinical decision-making ${ }^{3-6}$ and interpersonal communication. ${ }^{7}$ Mitigating clinician bias through targeted training in bias awareness and interpersonal communication skills is a promising strategy for decreasing healthcare disparities for racially, ethnically, and socio-economically disadvantaged persons. ${ }^{8}$ Few rigorously designed trials of communication skills target implicit bias as a factor contributing to health disparities. ${ }^{9,10}$ 
To address this gap, we designed a theoretically-grounded, multi-component, training intervention trial called "COmmuNity-engaged SimULation Training for Blood Pressure Control" (CONSULT-BP). The primary aim of the trial is to improve patient outcomes. The training program combines knowledge acquisition, bias awareness development, and simulation-based communication practice with racially and ethnically diverse patients in order to better prepare clinicians for patient interactions in which implicit bias may adversely affect healthcare outcomes. The clinical focus of the intervention is hypertension management, as it represents a challenging public health priority ${ }^{11}$ with persistent healthcare disparities and clinical outcomes mediated in part by the quality of clinician-patient communication and implicit bias as a contributing factor. ${ }^{12}$

A unique feature of this trial is the use of participatory action research (PAR) for training program development, implementation and modification. ${ }^{13}$ PAR is an implementation research methodology used to locally adapt evidence-based interventions tested in limited, controlled settings, to best meet the needs of stakeholders, with applicability more broadly beyond the local setting.

The purpose of this report is to describe the PAR design-strategy, educational intervention, and clinical trial methodology. We highlight early results-driven intervention revisions resulting from our use of the PAR-based strategy. We summarize key elements of using PAR in the clinical trial with particular attention to how its collaborative, reflective approach allows for a complex adaptive system to refine core training program components without compromising intervention content and study design. This report provides a resource for others seeking to implement training interventions that mitigate implicit bias in clinicianpatient encounters.

\section{Methods}

\section{The CONSULT-BP Educational Intervention}

\section{Core Educational Elements}

The core elements of the educational intervention are: 1. National Institute of Minority Health and Health Disparities-funded e-learning modules to build knowledge about health disparities, implicit bias, and patient-centered communication skills; ${ }^{14}$ 2. Implicit Association Tests (IAT) ${ }^{15}$ with results feedback to develop personal bias awareness, along with strategies to mitigate bias developed by Devine et $\mathrm{al}^{16} ; 3$. high-fidelity simulated clinical encounters with standardized patients (SPs) from the community for skills practice ${ }^{17}$; and 4 . evidence-based practice knowledge about hypertension management. We focused on implicit bias given the evidence of its impact on interpersonal communication ${ }^{7}$ and patient outcomes, ${ }^{2,5}$ particularly in hypertension where it contributes to a complex interaction of suboptimal clinical decision making and unsatisfactory patient experience of care. ${ }^{18}$ (Figure 1)

\section{Theoretical Framework}


The educational intervention and its delivery were designed to reflect key features of an adaptation of Bennett's intercultural competency framework. ${ }^{19,20}$ (Figure 2) In this theoretical model, to overcome denial about ones' own implicit biases, learners need to first acquire knowledge and understanding about implicit bias. Then, to help learners develop acceptance of the effect of implicit bias on healthcare disparities, learners need to move toward integration and recognition of implicit bias within themselves and their clinical encounters. The ultimate goal is to motivate learners to acquire and apply effective skills in situations where implicit bias is likely to arise.

Applying the simulation-based model of repeated practice and feedback for skills acquisition and progression to skill mastery ${ }^{21}$, the CONSULT training featured "mock" clinical encounters designed to "activate" trainee biases in the clinical care setting. To replicate the "authentic" experience of implicit bias in clinical care, the CONSULT intervention developed face-to-face simulated clinical encounters with "acting" SPs recruited from local racial and ethnic communities as a foundational component of the program. These face-to-face interactions provided a "contact-based educational intervention." ${ }^{20}$ Contact with groups for which one may hold biased attitudes may help reduce such bias. ${ }^{22,23}$ For those diverse individuals participating as trained CONSULT SP's, the simulation intervention created an opportunity for empowerment and equity, as community SP's provided direct skills feedback to healthcare trainees and contributed their "voice" as equal partners in the team effort to develop and refine the simulation scenarios. We sought to understand whether this creative and novel approach to communication skills training of healthcare professionals catalyzed the motivation of our learners to take their patient communication skills to a higher level of mastery through direct, objective, and specific feedback from individuals of color trained as SPs. ${ }^{20,24,25}$

\section{PAR Stakeholder Engaggement}

To adapt the core educational elements and strategies into a feasible and acceptable educational intervention, we used a PAR approach in which investigators collaboratively partnered with stakeholder participants. The goal was to work together to address system-specific issues affecting program operationalization. ${ }^{13}$ Community stakeholder partners for educational design and delivery were $\mathrm{racial} / \mathrm{ethnic}$ and socioeconomically diverse community leaders representing our local patient population. A local community health organization served as the community member recruitment liaison. Key academic stakeholder partners for educational design were School of Medicine and Graduate School of Nursing faculty from our target healthcare system. A separate community-based transformational change organization, with extensive experience in staff bias training, was engaged to help design inperson facilitation around implicit bias. These partners worked together to design case simulation scenarios, a trainee performance evaluation checklist, and SP training protocols that were integrated into a cohesive, replicable training program. Community advisors also assisted with recruiting community members representing the demographics of the medical center's local service population to work as community SPs. During the design process, we recruited faculty and trainees from the participating 
clinical training programs to pilot test the program in order to assess acceptability and clinical training relevance.

\section{Structure and Delivery of Educational Program - CONSULT 1.0}

Our stakeholder-engaged design process resulted in an intervention that applies a blended learning format to deliver information about implicit bias, communication skills, and hypertension management via online modules, all of which precede clinical simulation practice. At the recommendation of faculty stakeholders, program delivery occurred over two, in-person sessions, five weeks apart, to leverage a spaced learning design. ${ }^{26}$ The sessions were designed to minimize the time burden of the intervention on trainees outside of the classroom. As such, the in-person training sessions combined multiple components that trainees completed individually, but as part of an onsite group session. In each educational session, components included: "individual" online didactic modules, online IATs, face-to-face clinical practice simulation with SPs; and "group-based" facilitated debriefing sessions. Skills to mitigate the effects of implicit bias detailed by Devine et al ${ }^{16}$ were addressed as part of the group-based IAT debriefings. Each of the two, in-person sessions lasted three to four hours.

\section{Trial Design}

The educational intervention was integrated into the residency programs in Internal Medicine (IM) and Family Medicine (FM), and into the curriculum for Doctor of Nursing Practice (DNP) students at our institution. The impact of the CONSULT-BP training model is being tested through a stepped-wedge cluster randomized trial. This trial design allows for all trainees in any given year to be assigned to the intervention, which was a pre-condition for training program participation. The training intervention is a program requirement. This design is statistically advantageous as all trainees have both control and intervention periods, such that each individual serves as his/her own control, faciliating comparisons both within and across participants. Our model randomizes training times to one of five start dates within each academic year to accommodate pre-existing training schedules and to mitigate the effect of temporal trends in clinical skill proficiency.

\section{Trainee Enrollment and Data Collection}

Training programs assign their own trainees to participate in the intervention. Eligibility criteria for inclusion of trainee measures in the trial's outcomes analysis are: 1. practice at a clinical site supported by the medical center's electronic medical record (EMR) to allow data collection for BP outcome measurement; 2. a 10-week clinical look-back period; and 3. no prior completion of the CONSULT-BP intervention. Trainees are provided a fact sheet and asked to opt-out of the study if they do not want their data used for outcomes analysis.

\section{$\underline{\text { Trainee Measures }}$}


Trainee Implicit Association Tests (IAT), Explicit Bias Measures, Bias Awareness, and Reaction to the IAT. In addition to completing online Race/Ethnicity IATs [Black/White, Latino/White] ${ }^{36}$ and Race/EthnicityMedical Compliance IATs [Black/White, Latino/White] ${ }^{37}$, corresponding explicit questions are presented as part of the online intervention. Trainees are asked about their own explicit beliefs and perceptions of what "other health professionals" believe about race/ethnicity and race/ethnicity-related medical compliance in order to assess trainees' perception of their own bias as being "better than average". ${ }^{27}$ To assess trainees' reaction to the IAT, three questions are included from Howell \& Ratliff, 2016, ${ }^{27}$ using a 4point scale (Strongly disagree, Disagree, Agree, Strongly Agree) to measure the trainees' degree of defensiveness to the IAT. Trainees also complete a 7-item Bias Awareness Scale, with items assessed on a 6-point scale (strongly agree to strongly disagree) and higher scores indicating greater bias awareness. $^{28}$

Trainee Assessments by SPs. Community SPs complete standardized checklists of trainee performance measuring communication skills, emotional response/concern, BP measurement technique, and global performance.

\section{Patient Measures}

The primary trial outcome is the change in BP as reported in the EMR. Secondary outcomes include selfreported adherence to visits, diet modification and antihypertensive medication use as measured by the BP Self-Care Scale, ${ }^{29}$ and quality of communication and trust measured in the Health Care Climate Questionnaire ${ }^{30}$ and the trust sub-scale of the Primary Care Assessment Survey. ${ }^{31}$ These surveys are administered in clinic offices of participating trainees in the 10-weeks before and after the educational intervention. Following the stepped wedge design, all comparisons are before and after the intervention within patients nested within trainee and across trainees.

\section{Qualitative Feedback about the Educational Program}

We planned for ongoing early feedback from community SPs and faculty through periodic informal debriefing sessions, and from trainees via formal post-program online surveys. We also captured realtime observations and comments in fieldnotes maintained by research staff. Further, we conducted formal, individualized feedback meetings with groups of trainee representatives from the IM, FM and DNP programs, CONSULT-BP program faculty, and community SPs in June and July 2019. Invitations for individualized feedback was extended to all program faculty and SPs, and the 125 trainees who completed the first-year CONSULT-BP program. A Masters' level educational curriculum specialist conducted all feedback sessions and took extensive fieldnotes to capture respondents' comments.

The study protocol was reviewed and approved by the University of Massachusetts Medical School Institutional Review Board (IRB).

\section{Preliminary Results}


For pilot testing, we recruited DNP students, MD/PhD students in the research phase of training, residency and DNP program and simulation lab faculty. Because of scheduling demands, we were unable to recruit trainees from either participating residency. Pilot testing elucidated the need for clear, skilled facilitation of the IAT result debriefing discussion, and detailed instructions for how to guide trainees through onsite sessions and simulation. Second round pilot testing evaluated the acceptability of the IAT debriefings. For the first cohort, 125 trainees were assigned to the CONSULT educational program, 97 completed both sessions, and 64 (51\%) enrolled in the trial.

\section{Stakeholder Feedback: CONSULT 1.0}

Fieldnote summaries, feedback from trainees during the onsite, group educational sessions and informant interviews with learners revealed that the online delivery of knowledge content was suboptimal because learners could choose not to engage with the material. For example, trainees who saw themselves as having no implicit bias issues could skim through the content. Furthermore, asking individuals to complete online modules in a group setting generated a perception of real-time peer pressure to speed through the online modules. In particular, learners of color reflected that they felt "pressured" to rush through the online modules given that their non-person-of-color peers were "racing through the content" and "calling it useless." As a result, some learners-of-color expressed strongly the need to create a "safe" setting in which to address implicit bias issues within their educational programs. Learners also recommended changing the delivery of knowledge content, and particularly patientcentered communication skills, to an in-person, small group, interactive session to promote relational skill-building through direct interpersonal contact among group participants.

Feedback about IAT completion in the group setting revealed further challenges. Learners reflected that receiving instantaneous IAT results could trigger feelings of disbelief that required time and safe space to process. While the program provided a faculty-led IAT debriefing that included discussion and videos about skills to mitigate bias, ${ }^{16}$ faculty facilitators expressed that these sessions were too abstract and not clinically relevant. Learners further noted that the IAT debriefing sessions lacked sufficient time for learners to integrate bias mitigation skills before engaging in the SP clinical simulation encounters immediately afterward.

Skilled facilitation was crucial to the learner experience, but the facilitation skills of the clinicianeducators leading the IAT debriefings were variable, particularly in their ability to effectively facilitate safe and meaningful discussions around race and bias. The most well-received facilitators were clinical psychologists whose training skills promoted effective group-based processing and normalization of emotionally challenging content such as racism. Both learners and facilitators recommended augmenting facilitators' skills with a specific focus on creating a safe space, and effectively facilitating participant interaction and comfort. Essential recommended facilitation skills include, for example, how 
to support and not tokenize learners of color while not being reluctant to challenge defensiveness and White privilege.

Tailoring CONSULT's educational content to each training program emerged as an important consideration. Building a 'one size fits all' program for all learners resulted in learner feedback that content should be relevant and customized to both the learners' program and training level in order to eliminate redundancy. For example, the DNP program had ample existing lectures on social determinants of health and the FM program had ample communication-skills building sessions with behavioral health educators.

Refinement of the Educational Intervention: CONSULT 2.0

Within the rubric of the PAR model, we used feedback to refine the CONSULT educational intervention without forfeiting core educational elements. We continued to emphasize core elements of knowledge, bias awareness, and skill-building practice, and continued spaced-learning design with two sessions over a 5-week period. The resulting refinements modified the program structure to be more efficient and simpler, saving both time and effort for faculty and learners alike while preserving the same overarching goal of acquiring and practicing skills to mitigate implicit bias in patient-clinician encounters. (Table 1)

Knowledge of Disparities, Bias and Hypertension Management

CONSULT 2.0 restructures the content of the two training sessions to be delivered predominantly inperson. Session one focuses on knowledge-building didactics that include slide presentations, readings and handouts about healthcare disparities, and adds an experiential exercise in which learners actively participate in learning how implicit bias operates that was originally in the IAT debriefing in CONSULT 1.0. This session is delivered by a multidisciplinary team that include clinician-educators, who deliver healthcare disparities content, and clinical psychologists, who introduce bias and racism and facilitate the experiential exercise. Hypertension management content is delivered either in-person by a clinician or via online learning at the discretion of the training program.

\section{Developing Awareness of Personal Bias}

To address the learning objective of raising personal awareness of bias, we retained the IAT component of the intervention in CONSULT 2.0, but with modifications. We reduced the number of IATs from four to one overall to reduce time, perceived redundancy, and to minimize defensiveness. We shifted the completion of the IATs from in-person, group learning sessions to individualized sessions completed on the learner's own time. Learners are provided with their IAT results online, in real time, after completion of the test, and are provided the standard IAT debrief to help explain results.

(https://implicit.harvard.edu/implicit/faqs.html) CONSULT staff is made available to discuss IATs at the learner's discretion.

Learning and practicing skills to mitigate bias simulated clinical encounters 
In CONULT 2.0's session two, the delivery of Devine's skills to mitigate bias ${ }^{16}$ is modified to be presented in a framework anchored by a mnemonic proposed by Cooper $^{32}$ - RELATE (Respect, Empathize, Listen, Ask, Ialk, Engage). Devine's skills were originally presented in CONSULT 1.0 in5the context of groupbased debriefings of trainees' IAT results. SPs provide learners with 1:1 directed feedback on the patientcenteredness of the encounter. In this way, the experiential skill-building exercise with SP's promotes an individualized learning experience allowing learners to self-assess and improve their soft skills in, for example, relationship building and interpersonal dynamics that convey empathy, active listening, and non-judgmental attitudes.

Finally, the engagement of clinical psychologists in CONSULT 2.0's sessions was expanded from just the post-SP encounter debriefs to active participation in the entire program, including the knowledge-based didactic component (session one) and skills building component (session two). The expanded role promotes trust-building between trainees and facilitators, and augments the quality and consistency of group facilitation. This results in "safe space" to foster learner trust and comfort in examining individual learning needs and to take steps to integrate and adapt to feedback, reflect on self-awareness, and progress from denial and defensiveness to adaptation and acceptance, as suggested by Bennett. ${ }^{19}$

\section{Discussion}

In approaching the task of implicit bias training in healthcare, a gap exists between what is theoretically known to be effective $\mathrm{e}^{21,33}$ and what is actually delivered in the course of program implementation. Appreciation of evidence that implicit bias exists in healthcare professionals has arguably been one of the most challenging aspects of addressing the persistent gaps in healthcare disparities. As modeled by Bennett and others, ${ }^{19}$ recognition and self-awareness of bias are the critical first steps in implicit bias training. Building on these priorities, the CONSULT program specifically targets these first stages in the learning process of bias mitigation for healthcare professions. Our initial experience and preliminary outcomes offer pragmatic insights into how to best design interventions to equip healthcare professionals with skills training in bias awareness and mitigation. The impact of this educational program on patient outcomes remains to be seen as our ongoing trial continues.

We leveraged the collaborative, cyclical, reflective inquiry design of the PAR approach to enable refinement of our educational intervention. In this way, we were able to enhance curricular implementation of IAT assessments; methodologic approaches to bias recognition, self-awareness and acceptance; and communication skills practice in SPs scenarios designed to trigger intrinsic bias. The modifications in CONSULT 2.0 addressed key stakeholder recommendations highlighting important "operational" features of "acceptability" to satisfy both trainees and faculty. These include reduced time burden; ensuring a "safe space" for teaching and learning about bias; developing face-to-face learning formats that foster relational engagement and individualized attention to learners in a small group setting; and engagement of expert facilitators, typically from clinical psychology, in the delivery of content and hands-on skills building in bias self-awareness and bias mitigation. 
To best meet the overarching goal of the CONSULT educational program, the CONSULT 2.0 intervention was explicitly redesigned to be a formative experience in which each learner can safely practice and refine their communication and relationship building skills as part of an ongoing path to mastery. The importance of recruiting and retaining highly skilled facilitators as part of this training process cannot be overstated. For this study, we were fortunate to have access to clinical psychologists who became deeply engaged as facilitators after we recognized the limited experience of most clinical faculty in managing the personally charged and highly sensitive issues regarding racism and personal perceptions of bias. This is not to say that this important work is solely dependent on the expertise of clinical psychologists, but rather to highlight that the facilitation skills for this type of training program requires proficiency in handling complex group dynamics, familiarity with trauma management skills, and experience in competency development models as articulated by Bennett, ${ }^{19}$ which feature the advancement of learner abilities from stages of denial and defensiveness to adaptation and acceptance. We learned that both cognitive and affective mastery of the material as well as the working knowledge and experience of highly-skilled clinical faculty were both essential for effective facilitation and learner training.

Our effort to implement theoretically sound and novel approaches to building clinician skills to mitigate bias and enhance patient-clinician communication across intercultural differences holds important implications for public health. While CONSULT-BP focuses on hypertension, the CONSULT model is readily adaptable to other diseases, such as COVID-19, in which decisions for testing are emerging as being subject to bias. ${ }^{34}$ This adaptability of the CONSULT model is an important feature as disparities in healthcare access and outcomes for racial, ethnic and poor populations is a growing problem spanning an increasing range of medical conditions and clinical services. Thus, the challenge of effectively addressing the adverse impact of bias through awareness building and bias mitigation skills, as recommended by the Centers for Disease Control and Prevention in the case of COVID-19, constitutes a pressing need public health. ${ }^{35}$ To date, the domain of educational interventions for bias training remains ripe for development and the preliminary findings from the CONSULT program contribute useful insights into the design of bias training models for healthcare professionals. Our study provides a roadmap for others who share these goals and offers lessons learned that others can use to advance their own efforts.

\section{List Of Abbreviations}

CONSULT-BP: COmmuNity-engaged SimULation Training for Blood Pressure Control

SPs: Standardized Patients

PAR: Participatory Action Research

IAT: Implicit Association Test

IM: Internal Medicine

FM: Family Medicine 
DNP: Doctor of Nursing Practice

EMR: Electronic Medical Record

\section{Declarations}

Ethics approval and consent to participate: Name of IRB Granting Institution: University of Massachusetts Medical School. Consent for all learners, patients, and standardized patients was collected in a written format using a consent form approved by the IRB.

Consent for publication: We have requested permission to reproduce the image in Figure 2 from Dr. Cayla Teal at Texas A \& M University

Availability of data and materials: Quantitative data are not yet available as data collection is in process.

Competing interests: The authors declare that they have no competing interests.

Funding: Research reported in this manuscript was supported by the National Institute of Minority Health and Health Disparities of the National Institutes of Health under award number R01MD011532. The funder had no role in the study design; in the collection, analysis and interpretation of data; in the writing of the report; and in the decision to submit the article for publication.

Authors' contributions: JT: conception and design of study, acquisition of study data, drafting and critically revising the manuscript; NE, JH, LR, JT, JC, GC, GP, VD, SS, AFS: Acquisition of study data, drafting and critically revising the manuscript; MP, OV, MG, MY, ED, EE, SP: conception and design of study, critically revising the manuscript; JS: conception and design of study, drafting and critically revising the manuscript. All authors have read and approved the manuscript.

Acknowledgements: We would like to thank the community advisors, standardized patients, and trainees for their generous contributions to this project.

\section{References}

1. Maina IW, Belton TD, Ginzberg S, Singh A, Johnson TJ. A decade of studying implicit racial/ethnic bias in healthcare providers using the implicit association test. Soc Sci Med. 2018;199:219-29.

2. FitzGerald C, Hurst S. Implicit bias in healthcare professionals: a systematic review. BMC Med Ethics. 2017;18(1):19.

3. Green AR, Carney DR, Pallin DJ, et al. Implicit bias among physicians and its prediction of thrombolysis decisions for black and white patients. J Gen Intern Med. 2007;22(9):1231-8.

4. Penner LA, Dovidio JF, West TV, Gaertner SL, Albrech TL. Aversive racism and medical interactions with black patients: A field study. J Exp Psychol. 2010;46(2):436-40. 
5. Sabin JA, Greenwald AG. The influence of implicit bias on treatment recommendations for 4 common pediatric conditions: pain, urinary tract infection, attention deficit hyperactivity disorder, and asthma. Am J Pub Health. 2012;102(5):988-95.

6. Bogart LM, Catz SL, Kelley JA, Benotsch EG. Factors influencing physicians' judgments of adherence and treatment decisions for patients with HIV disease. Med Decis Mak. 2001;21(1):28-36.

7. Cooper LA, Roter DL, Carson KA, et al. The associations of clinicians' implicit attitudes about race with medical visit communication and patient ratings of interpersonal care. Am J Pub Health. 2012;102(5):979-87.

8. Smedley BD, Stith AY, Nelson AR. Unequal treatment: Confronting racial and ethnic disparities in health care. Washington, D.C.: National Academy Press; 2003.

9. Beach MC, Gary TL, Price EG, et al. Improving health care quality for racial/ethnic minorities: a systematic review of the best evidence regarding provider and organization interventions. BMC Public Health. 2006;6:104.

10. Cooper LA, Roter DL, Bone LR, et al. A randomized controlled trial of interventions to enhance patientphysician partnership, patient adherence and high blood pressure control among ethnic minorities and poor persons: study protocol NCT00123045. Implement Sci. 2009;4:7.

11. Fisher NDL, Curfman G. Hypertension-A Public Health Challenge of Global Proportions. JAMA. 2018;320(17):1757-9.

12. Schoenthaler A, Chaplin WF, Allegrante JP, et al. Provider communication effects medication adherence in hypertensive African Americans. Patient Educ Couns. 2009;75(2):185-91.

13. Leykum LK, Pugh JA, Lanham HJ, Harmon J, McDaniel RR Jr. Implementation research design: integrating participatory action research into randomized controlled trials. Implement Sci. 2009;4:69.

14. Van Schaik E, Howson A, Sabin J. Healthcare Disparities. 2014(9675). .

15. Greenwald AG, McGhee DE, Schwartz JL. Measuring individual differences in implicit cognition: The Implicit Association Test. J Pers Soc Psychol. 1998;74(6):1464-80.

16. Devine PG, Forscher PS, Austin AJ, Cox WTL. Long-term reduction in implicit race bias: A prejudice habit-breaking intervention. J Exp Soc Psychol. 2012;48(6):1267-78.

17. Ziv A, Wolpe PR, Small SD, Glick S. Simulation-based medical education: an ethical imperative. Simul Healthc. 2006;1(4):252-6.

18. Hanlin RB, Asif IM, Wozniak G, et al. Measure Accurately, Act Rapidly, and Partner With Patients (MAP) improves hypertension control in medically underserved patients: Care Coordination Institute and American Medical Association Hypertension Control Project Pilot Study results. J Clin Hypertens (Greenwich). 2018;20(1):79-87.

19. Bennett MJ. A developmental approach to training for intercultural sensitivity. Int J Intercult Rel. 1986;10(2):179-96.

20. Teal CR, Gill AC, Green AR, Crandall S. Helping medical learners recognise and manage unconscious bias toward certain patient groups. Med Educ. 2012;46(1):80-8. 
21. Shute VJ, Gawlick LA. Practice Effects on Skill Acquisition, Learning Outcome, Retention, and Sensitivity to Relearning. Human Factors. 1995;37(4):781-803.

22. Dasgupta N, Greenwald AG. On the malleability of automatic attitudes: Combating automatic prejudice with images of admired and disliked individuals. J Pers Soc Psychol. 2001;81(5):1-15.

23. Fiske $S$. What we know about bias and intergroup conflict, the problem of the century. Curr Dir Psychol Sci. 2002;11(4):123-8.

24. Johnson RL, Roter DL, Powe NR, Cooper LA. Patient race/ethnicity and quality of patient-physician communication during medical visits. Am J Pub Health. 2004;94(12):2084-90.

25. Morell VW, Sharp PC, Crandall SJ. Creating student awareness to improve cultural competence: creating the critical incident. Med Teach. 2002;24(5):532-4.

26. Kerfoot BP, Fu Y, Baker H, Connelly D, Ritchey ML, Genega EM. Online spaced education generates transfer and improves long-term retention of diagnostic skills: a randomized controlled trial. J Am Coll Surg. 2010;211(3):331-7 e331.

27. Howell JL, Ratliff KA. Not your average bigot: The better-than-average effect and defensive responding to Implicit Association Test feedback. Br J Soc Psychol. 2017;56(1):125-45.

28. Girod S, Fassiotto M, Grewal D, et al. Reducing Implicit Gender Leadership Bias in Academic Medicine With an Educational Intervention. Acad Med. 2016.

29. Peters RM, Templin TN. Measuring blood pressure knowledge and self-care behaviors of African Americans. Res Nurs Health. 2008;31(6):543-52.

30. Kasser VG, Ryan RM. The Relation of Psychological Needs for Autonomy and Relatedness to Vitality, Well-Being, and Mortality in a Nursing Home. J Appl Soc Psychol. 1999;29(5):935-54.

31. Safran DG, Kosinski M, Tarlov AR, et al. The Primary Care Assessment Survey: tests of data quality and measurement performance. Med Care. 1998;36(5):728-39.

32. Cooper L. Doctors can fight unconscious racial bias: A simple checklist would remind physicians to treat patients of all races equitably. 2018. https://www.bloomberg.com/opinion/articles/2018-0828/doctors-can-fight-implicit-bias-against-african-american-patients. Published August 28, 2018. Accessed on June 16, 2020.

33. Sukhera J, Watling C. A Framework for Integrating Implicit Bias Recognition Into Health Professions Education. Acad Med. 2018;93(1):35-40.

34. Rubix Life Sciences. COVID-19 and Minority Health Access.; 2020. https://rubixls.com/wpcontent/uploads/2020/04/COVID-19-Minority-Health-Access-7-1.pdf. Accessed May 21, 2020.

35. Eligon J, Burch A. Questions of Bias in COVID-19 Treatment Add to the Mourning for Black Families. The New York Times. https://www.nytimes.com/2020/05/10/us/coronavirus-african-americansbias.html. Published May 10, 2020. Accessed on May 21, 2020.

\section{Tables}


Table 1. COmmuNity-engaged SimULation Training for Blood Pressure Control

(CONSULT-BP): Core elements and Tailored Elements

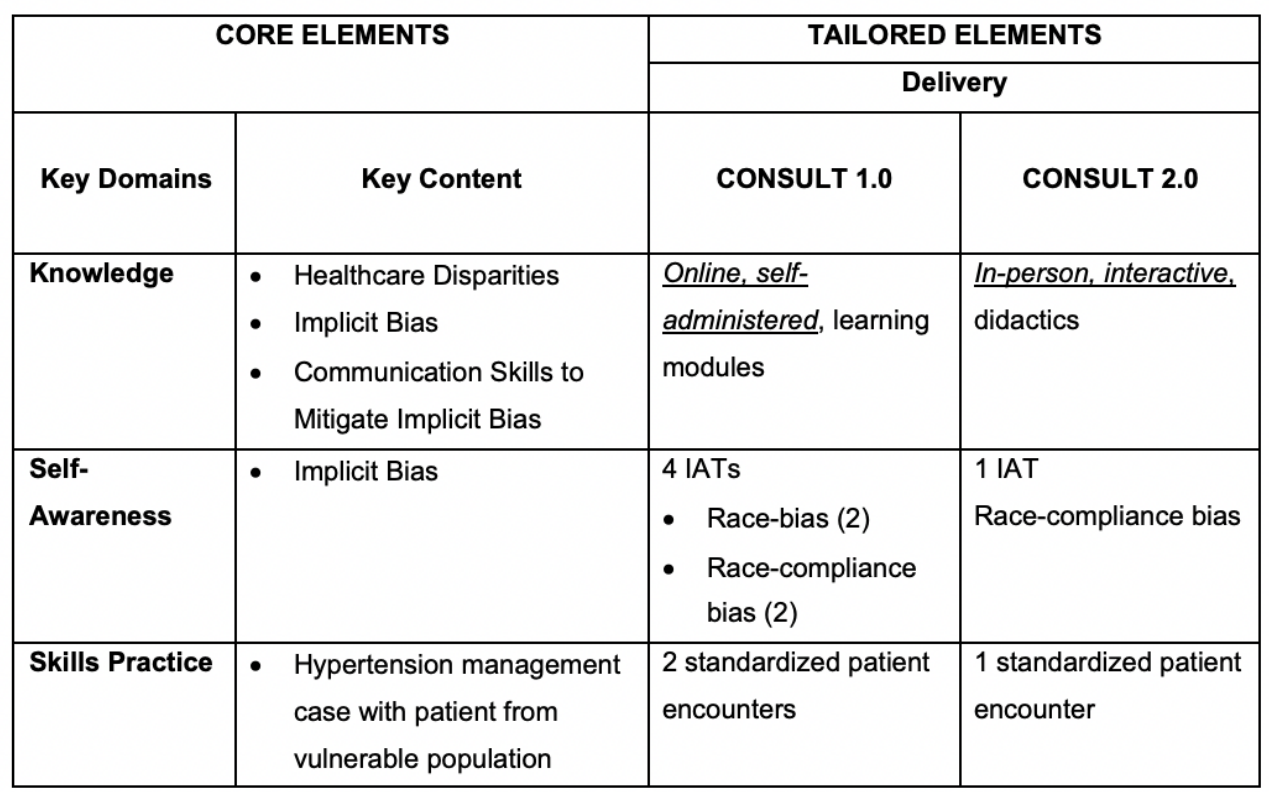

IAT = Implicit Association Test

\section{Figures}




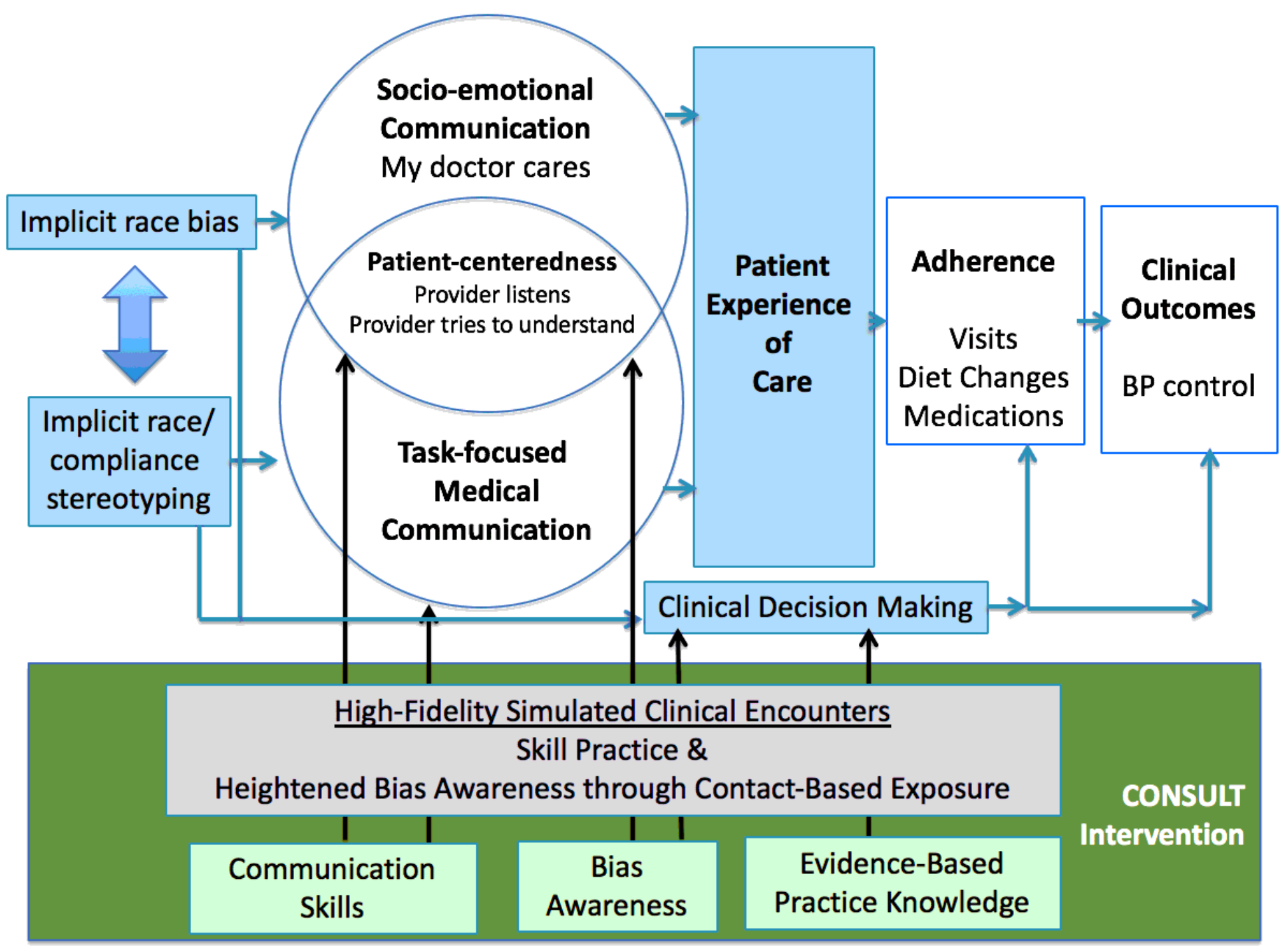

Figure 1

The CONSULT-BP intervention model 


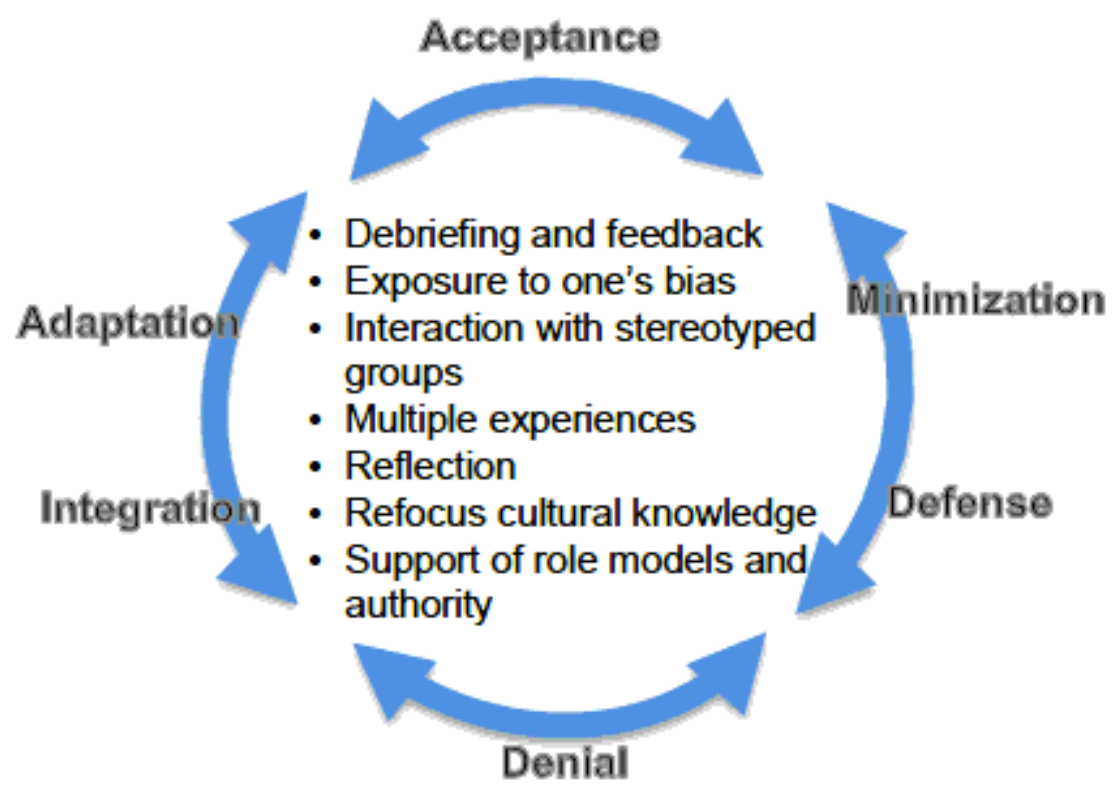

Figure 2

Adaptation of Bennett's intercultural competency theoretical framework19,20 